\title{
Intestinal absorption of two dipeptides in Hartnup disease'
}

\author{
A. M. ASATOOR, B. CHENG, K. D. G. EDWARDS, A. F. LANT, \\ D. M. MATtheWS, M. D. Milne, F. NAVAB, AND A. J. RiChaRdS \\ From the Medical Unit and Department of Chemical Pathology, Westminster Medical School, London
}

SUMMARY The results of oral tolerance tests of two dipeptides and of their constituent amino acids are compared in normal subjects and in a case of Hartnup disease. In the control subjects the rate of absorption of phenylalanine from phenylalanyl-phenylalanine and of tryptophan from glycyl-tryptophan was slower than after the equivalent amount of the free amino acids. Absorption of the two essential amino acids (tryptophan and phenylalanine) in the patient was almost zero after administration in the free form, but was much greater after the dipeptide.

Results of experiments on absorption and hydrolysis of the two peptides in the rat small intestine are also reported.

It is suggested that whereas normal subjects absorb essential amino acids by a dual mechanism of mucosal uptake of free amino acids and oligopeptides, nutrition in Hartnup disease is largely dependent on uptake of oligopeptides containing the amino acids affected by the intestinal transport defect of the disease.

Recent investigations have emphasized the importance of a dual mechanism for intestinal absorption of protein digestion products (Newey and Smyth, 1962; Craft, Geddes, Hyde, Wise, and Matthews, 1968; Matthews, Lis, Cheng, and Crampton, 1969), involving (a) transport of free amino acids from the gut lumen, and (b) uptake of oligopeptides followed (with a few possible exceptions) by hydrolysis to the constituent amino acids by oligopeptidases within, or at the brush border of, the intestinal cell. The relative importance of the two modes of absorption is not yet determined. In Hartnup disease, the former mechanism for many neutral amino acids is grossly inadequate although not entirely absent (Milne, Crawford, Girao, and Loughridge, 1960; Navab and Asatoor, 1970), whereas oligopeptide absorption may be either normal or only slightly reduced (Navab and

'Address for correspondence: Professor M. D. Milne, Medical Unit, Westminster Hospital, 17 Page Street, London, SW1.

Received for publication 2 April 1970.
Asatoor, 1970). The disease, therefore, provides an almost unique opportunity for the study of the importance of intestinal absorption of oligopeptides in man. This paper describes tests of absorption of two dipeptides and of the corresponding amino acids in a patient suffering from Hartnup disease and in normal control subjects.

\section{Methods}

The methods used in this investigation were not ideal, but were conditioned by the following difficulties.

Oligopeptides are difficult to prepare in bulk and commercial samples are extremely expensive. Throughout the investigation, therefore, a compromise had to be adopted using the minimum dose of oligopeptide which would be expected to give an increase of plasma amino acids sufficient in amount to avoid appreciable experimental error. 
Increase of plasma amino acids during peptide or amino acid tolerance tests in Hartnup disease is usually less than in the normal subject, but obviously the difference cannot be accurately predicted before the investigation has been carried out. For this reason the patient was given larger doses than the controls.

Hartnup disease is extremely rare and only a single patient was available for study. Although she was very cooperative, only a limited number of tolerance tests could be performed.

Previous investigations in the same patient (Navab and Asatoor, 1970) had shown that the dipeptide carnosine ( $\beta$-alanyl-L-histidine) was absorbed normally. By contrast, when an equivalent mixture of the constituent free amino acids was given by mouth, absorption of histidine was grossly impaired despite completely normal $\beta$-alanine absorption. The patient agreed to further tolerance tests with two other dipeptides and the corresponding amino acids. Obviously, the peptide selected had to contain either one or two of the amino acids known to be poorly absorbed in Hartnup disease. The previous peptide investigated contained only a single affected amino acid, namely, histidine, and therefore the first peptide, L-phenylalanyl-Lphenylalanine (phe-phe), was selected to contain two such amino acids. A tryptophan-containing peptide, glycyl-tryptophan (gly-trp), was selected as the second one to be investigated, because of the disproportionate evidence of tryptophan deficiency in Hartnup disease. Tests of possible toxicity were performed before the dipeptides were given to the patient, both in normal human volunteers (some of the authors of this paper), and at five times the dosage per kilogram body weight in the rat. No such toxicity was found to occur.

\section{INVESTIGATIONS IN MAN}

\section{Phenylalanyl-phenylalanine (phe-phe)}

Oral tolerance tests of the dipeptide and of the equivalent amount of L-phenylalanine (which would be produced from the peptide by hydrolysis) were carried out in the morning fasting state. Venous blood samples were obtained before ingestion of either the peptide or of the free amino acid and at 15, 30, 45, 60, 90 , and 120 minutes thereafter. Dosage of phenylalanine in the patient was $0.60 \mathrm{~m}-\mathrm{mole} / \mathrm{kg}$ body weight, and of phenylalanyl-phenylalanine $0.30 \mathrm{~m}$-mole $/ \mathrm{kg}$ body weight, each being taken in $500 \mathrm{ml}$ flavoured water. Similar tests were carried out in two normal subjects, but here the dosage was $0.20 \mathrm{~m}$-mole $/ \mathrm{kg}$ body weight of phenylalanine, and $0 \cdot 10 \mathrm{~m}$-mole $/ \mathrm{kg}$ body weight of the dipeptide. The difference in dosage was entirely conditioned by limited supplies of the pure peptide.
Glycyl-L-tryptophan (gly-trp)

Similar tests were carried out, but an additional blood sample was taken at 180 minutes. Dosage in the patient was $0.09 \mathrm{~m}$-mole $/ \mathrm{kg}$ body weight of both the peptide and the mixture of the two constituent free amino acids. Dosage in five normal adult subjects was $0.05 \mathrm{~m}$-mole $/ \mathrm{kg}$ body weight. The difference in dose was conditioned by limited supplies of the dipeptide, and by the expectation, subsequently confirmed, that absorption would be reduced in Hartnup disease.

PARALLEL EXPERIMENTS IN RATS

\section{Glycyl-tryptophan}

Solutions of the dipeptide and of the two constituent amino acids were prepared in rat tyrode solution at a concentration of $0.025 \mathrm{~m}-\mathrm{mole} / \mathrm{ml}$. Male albino rats weighing about $300 \mathrm{~g}$ were lightly anaesthetized with ether, and a length of jejunum of approximately $10 \mathrm{~cm}$ in length was tied off by two separate ligatures, the proximal one being just distal to the entrance of the bile duct into the gut. Warm solutions of either the dipeptide or of the free amino acids were injected into the lumen of the segment at a dosage of $4 \mathrm{ml} / \mathrm{kg}$ body weight. The incision was sutured immediately after the injection, and the anaesthesia was maintained for 15 minutes. After this period the contents of the gut segment were rinsed out with distilled water, boiled for $5 \mathrm{sec}$, made up to volume, and precipitated protein was removed by filtration. The mucosa was scraped off the whole segment and immediately boiled in $1 \mathrm{ml}$ of distilled water. Immediately after excision of the gut segment, blood was obtained from the left ventricle in a heparinized syringe.

In two animals after the peptide solution had been within the segment for $15 \mathrm{~min}$, the contents were incubated for three hours in vitro at $37^{\circ} \mathrm{C}$, both with and without prior centrifugation. Aliquots for analysis were taken at 15-min intervals. These aliquots were immersed in a boiling water bath for $20 \mathrm{sec}$ to destroy peptidases before being made up to volume.

Similar experiments with phenylalanyl-phenylalanine were carried out, but in this case no estimations of the serum amino acids were made, and a fixed volume of $1 \mathrm{ml}$ of the dipeptide or phenylalanine solution was injected. This dipeptide is very insoluble and the injection was only possible as a finely ground suspension via a tied-in plastic cannula.

\section{ANALYTICAL METHODS}

\section{Phenylalanine in serum}

The Technicon amino acid analyser with the standard procedure described in the Technicon 
Handbook (Technicon Instrument Co., 1966) was used.

\section{Phenylalanine in fluid from gut lumen}

Separation was by thin-layer chromatography on MN Polygram Cel 300 (Camlab), using as developing solvent pyridine 10:methylethylketone 50 ; n-butanol 10: glacial acetic acid 2: water 20; colour development by ninhydrin ( $2 \%$ in acetone at $45^{\circ}$ for $\left.30 \mathrm{~min}\right)$; estimation by photometric scanning by reflected light (JoyceLoebl Chromoscan, filter 5040).

Tryptophan in serum and intestinal contents The colorimetric method of Udenfriend and Peterson (1957) was used.

\section{Glycine in serum and intestinal contents}

The method of Alexander, Landwehr, and Seligman (1945), modified by $2 \%$ ninhydrin solution instead of $1 \%$, was used. Peptides in intestinal contents were estimated by increase of glycine or of free phenylalanine after acid hydrolysis at $110^{\circ} \mathrm{C}$ in a sealed tube for 16 hours. Estimations of glycine, tryptophan, and glycyltryptophan in intestinal contents were confirmed by the quantitative chromatographic method of Young, Reid, and Edwards (1964), ethyl acetate

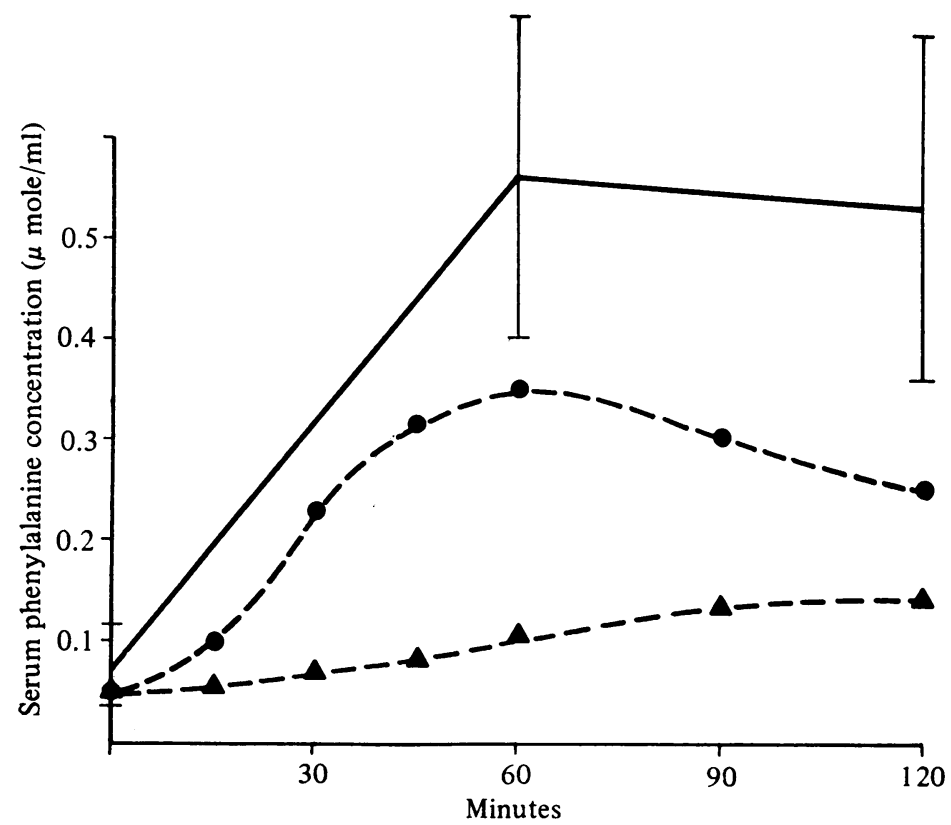

Fig. 1 Serum levels of phenylalanine during the first 120 min after ingestion of L-phenylalanine $(0.6 \mathrm{~m}$-mole/ kg body weight) in 19 normal subjects (continuous line), and in a case of Hartnup disease (broken line, triangles) and after phenylalanyl-phenylalanine $(0.3 \mathrm{~m}$-mole $/ \mathrm{kg}$ body weight) in the same patient (broken line, closed circles). The vertical lines in the normal subjects are 1 standard deviation from the mean. Data in the controls are taken from Hsia, Knox, and Paine (1957).
60 : pyridine 25 : water 20 being used as developing solvent.

\section{Results}

RESULTS IN MAN

L-phenylalanyl-L-phenylalanine (phe-phe)

No phenylalanyl-phenylalanine was detected in serum samples either in Hartnup disease or in the two normal subjects, the main change in serum amino acids being a large rise in phenylalanine and a smaller increase in tyrosine. Figure 1 gives serum levels of phenylalanine in the case of Hartnup disease after ingestion of the dipeptide and of the corresponding dose of free phenylalanine. The results are compared with values obtained from 19 normal subjects after ingestion of the same dose of phenylalanine (Hsia, Knox, and Paine, 1957). Serum concentrations of phenylalanine are grossly below normal values after ingestion of the free amino acid but are much higher after the oligopeptide. The range of the normal tolerance test after phenylalanyl-phenylalanine is naturally unknown, as current supplies of the peptide are inadequate for this investigation. The best index of total absorption is the area under the tolerance curve during the absorptive period. In the first 60 min the area in Hartnup disease after the dipeptide is 0.67 of the mean normal after phenylalanine, whereas the corresponding ratio in Hartnup disease after the amino acid is only $0 \cdot 13$. Therefore, absorption of phenylalanine by the patient was approximately five times as great after the dipeptide as after the free amino acid.

Owing to limitation of supply of the dipeptide, tolerance tests in the two normal subjects were at one third of the dosage shown in Fig. 1 and are, therefore, not comparable. In both subjects absorption was more rapid after the free amino acid than after the peptide (Fig. 2), the ratios of the areas under the tolerance curves for the first $60 \mathrm{~min}$ being 2.0 and 2.3 respectively. Serum levels of phenylalanine in subject 1 were considerably higher than those of subject 2 both after the amino acid and after the peptide, the ratios of the corresponding areas between the two subjects being $2 \cdot 1$ for phenylalanylphenylalanine and 1.8 for phenylalanine. The peak values of the curves after the dipeptide were considerably later than after the free amino acid.

Comparison of the results in the patient with those of the normals is somewhat speculative owing to the considerable difference in dosage. The area under the patient's tolerance curve for the 60 -min period after ingestion of the peptide 


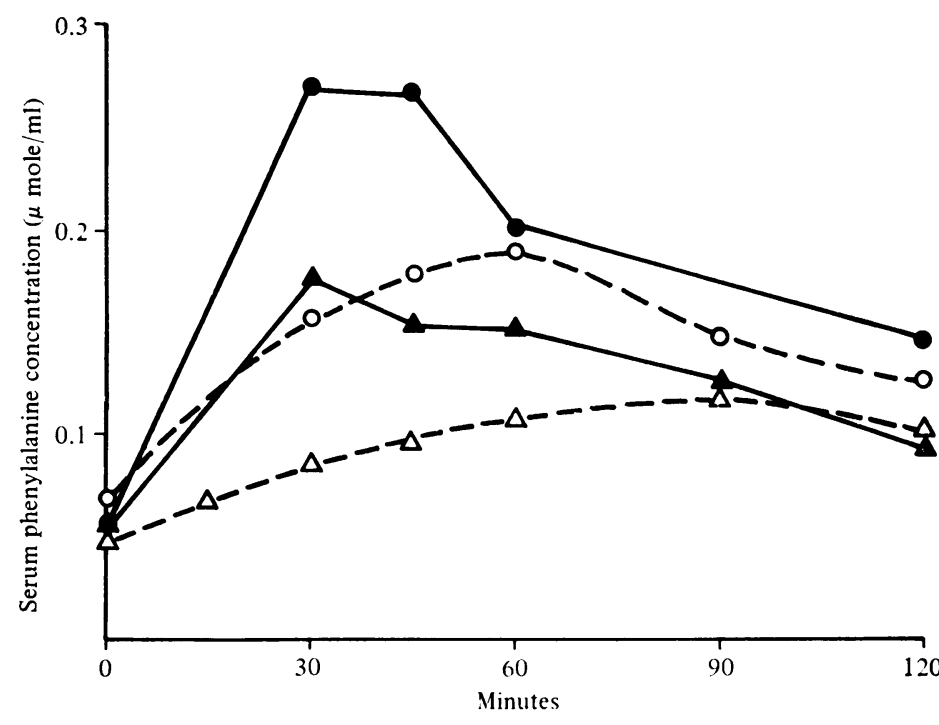

Fig. 2 Serum levels of phenylalanine in two normal subjects during the first 120 minutes after ingestion of L-phenylalanine ( $0 \cdot 2 \mathrm{~m}$-mole/ $\mathrm{kg}$ body weight $)$ shown as continuous lines, and after phenylalanyl-phenylalanine ( $0.1 \mathrm{~m}$-mole/kg body weight $)$ shown as broken lines. The values are higher after the free amino acid than after the peptide.

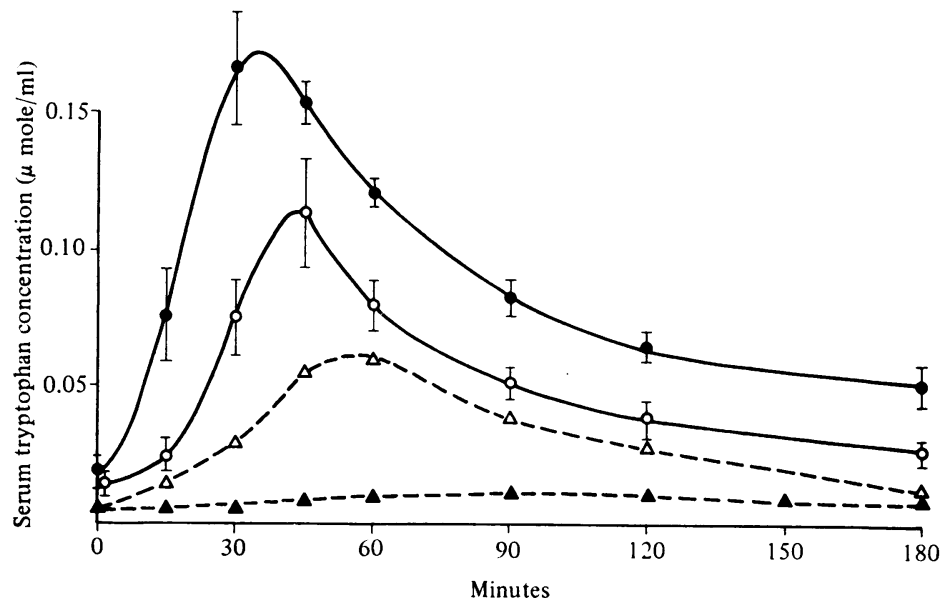

Fig. 3 Serum tryptophan concentrations during the first 180 minutes after ingestion of glycyl-tryptophan or the equivalent glycine and tryptophan mixture in five normal subjects (dosage $0.05 \mathrm{~m}$-mole/kg body weight) and a case of Hartnup disease (dosage $0.09 \mathrm{~m}$-mole/kg body weight).

Continuous line with closed circles represents normals after the amino acid mixture: continuous line with open circles normals after the dipeptide; broken line with open triangles Hartnup disease after the dipeptide; broken line with closed triangles Hartnup disease after the amino acid mixture.

The vertical lines give the standard error of the mean. was 2.9 that of the mean of the two normal subjects. As the dosage ratio was three to one, this suggests that absorption of phenylalanylphenylalanıne by the patient was probably within normal limits.

\section{Glycyl-L-tryptophan ( $g$ ly-trp)}

No glycyl-tryptophan was detected in serum samples either in Hartnup disease or in normal controls. Figure 3 gives serum concentrations of trp after ingestion of the dipeptide and of the equivalent mixture of glycine and tryptophan in five normal subjects and in the case of Hartnup disease. Again, the dosages are not comparable, the patient being given 1.8 times the dose in normal subjects. Concentrations of tryptophan are lower in the patient than in the normal subjects after the dipeptide, and the rise of serum tryptophan is almost zero after the glycine and tryptophan mixture. The relative areas under the tolerance curves between zero and $60 \mathrm{~min}$ are for the peptide $\mathbf{0 . 3 5}$ of the normal and for the free amino acid mixture 0.016 of the normal Allowing for the difference in dosage, absorption in the patient was about 0.20 of normal after glycyl-tryptophan, and 0.01 of normal after the amino acid mixture. Comparing the two tolerance tests in Hartnup disease without reference to the results in normal subjects, absorption was approximately 12 times as effective after the dipeptide as after the amino acid mixture. By contrast, in the normal subjects absorption was about 1.9 times better after the free amino acids than after glycyl-tryptophan. Statistical analysis, using the paired $t$ test, shows a significantly greater mean value for serum tryptophan in the normal subjects after the amino acid mixture than after the peptide. The paired $t$ test is valid since the same five individuals were compared in the two separate types of tolerance test.

The absorption of glycine, unlike that of tryptophan, is not grossly abnormal in Hartnup disease. Figure 4 gives serum glycine concentrations after ingestion of the peptide and of the amino acid mixture in normal subjects and in the case of Hartnup disease. Allowing for the higher dosage in the patient, the tolerance tests are not appreciably different, although the peak values are later in the patient than in the normals. An apparent anomaly is seen from the combined analysis of Figures 3 and 4 . If a dipeptide is absorbed entire, the serum increments of the two constituent amino acids, expressed as $\mu \mathrm{moles} / \mathrm{ml}$, should be approximately equal providing that their clearance rates from plasma are similar. This occurred in the normal subjects, where the mean maximal rise of serum tryptophan was $0.10 \mu \mathrm{mole} / \mathrm{ml}$ and of serum glycine $0.12 \mu \mathrm{mole} / \mathrm{ml}$. By contrast, in Hartnup disease, the figures for a larger dose of the dipeptide were $0.06 \mu \mathrm{mole} / \mathrm{ml}$ for serum tryptophan and $0.18 \mu \mathrm{mole} / \mathrm{ml}$ for serum glycine. The rat 


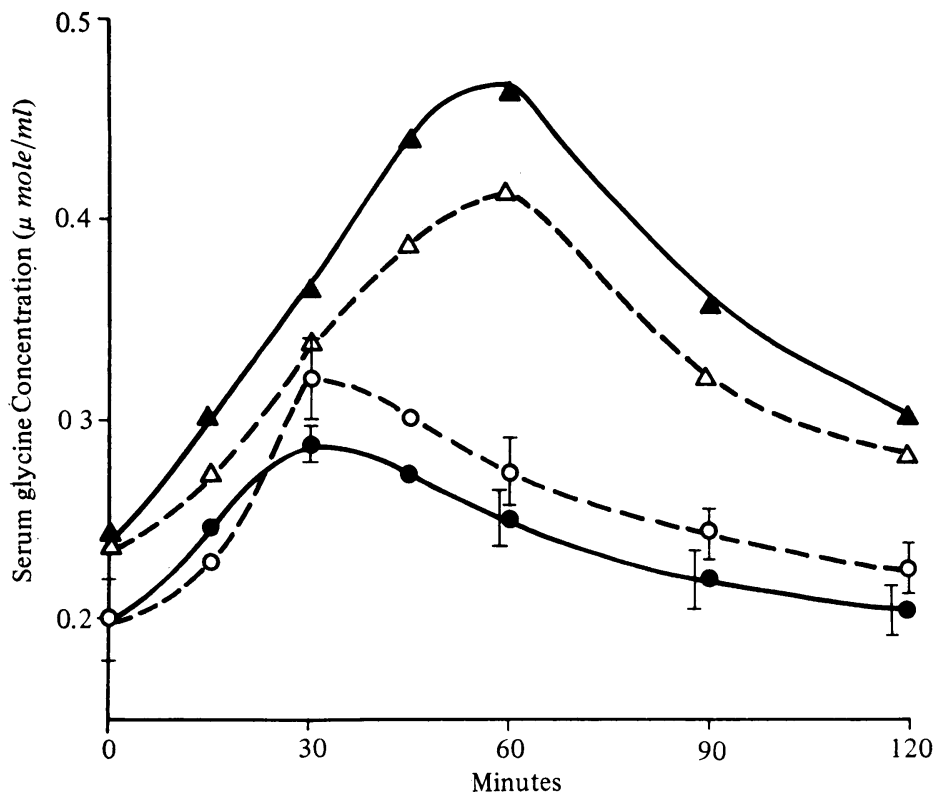

Fig. 4 Serum glycine concentrations after ingestion of glycyl-tryptophan or the equivalent amino acid mixture as in Figure 3.

Continuous line with closed triangles represents Hartnup disease-amino acid mixture; broken line with open triangles Hartnup disease-peptide; continuous line with closed circles controls-amino acid mixture; broken line with open circles controlspeptide.

The vertical lines give the standard error of the msan.

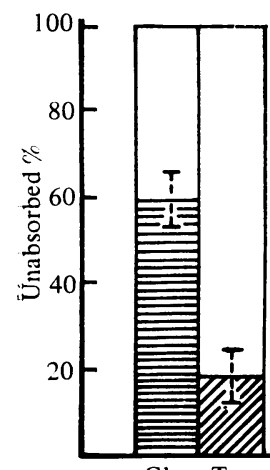

Gly Trp

(a)

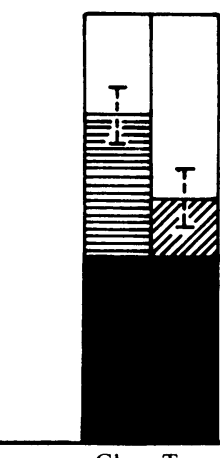

(b)

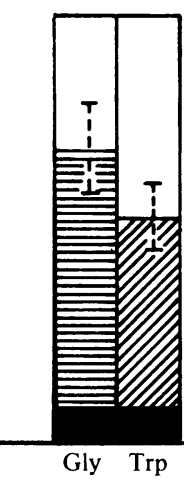

(c)

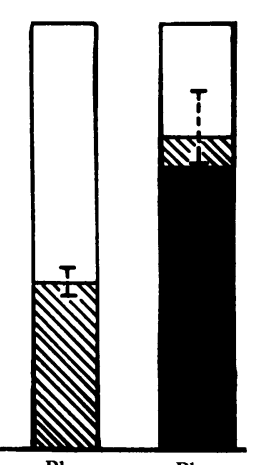

(d)
Phe

(e)
Fig. 5 Absorption of peptides or equivalent amino acids from gut segments of anaesthetized rats. The black column in each case gives the concentration of unabsorbed peptide and the shaded areas unabsorbed free amino acids.

From left to right:

a) Glycine tryptophan mixture $(0.025 \mathrm{~m}$-mole $/ \mathrm{ml})$

b) Glycyl tryptophan $(0.025 \mathrm{~m}$-mole $/ \mathrm{ml})$

c) Glycyl tryptophan $(0.010 \mathrm{~m}-\mathrm{mole} / \mathrm{ml})$

d) Phenylalanine $(0.05 \mathrm{~m}$-mole $/ \mathrm{ml})$

e) Phenylalanyl-phenylalanine $(0.025 \mathrm{~m}$-mole $/ \mathrm{ml})$

The vertical lines in $(a),(b)$, and $(c)$ give the standard error of the means, and in $(d)$ and $(e)$ the range of three estimations. experiments provide a probable explanation for the discrepancy by showing that large quantities of free tryptophan and free glycine appear within the gut lumen during the absorption of the dipeptide. Both of the liberated free amino acids would ultimately be absorbed by normal subjects, but in Hartnup disease little or no absorption of the liberated free tryptophan will occur.

\section{RESULTS IN RAT EXPERIMENTS}

Gly-trp

Serum concentrations of tryptophan were significantly higher after intestinal injection of the amino acid mixture than after the dipeptide, the values being, gly and trp mixture, $0.13 \pm$ $0.04 \mu \mathrm{mole} / \mathrm{ml}(\mathrm{n}=8)$ and gly-trp, $0.06 \pm 0.02$ $\mu \mathrm{mole} / \mathrm{ml}(\mathrm{n}=9) \mathrm{t}=3.0, \mathrm{P}<0.01$.

By contrast, serum glycine concentrations were not significantly different: gly and trp mixture, $0.57 \pm 0.07 \mu \mathrm{mole} / \mathrm{ml}(\mathrm{n}=8)$ and gly-trp, $0.63 \pm 0.07 \mu \mathrm{mole} / \mathrm{ml}(\mathrm{n}=9), \mathrm{t}=1.4, \mathrm{P}>$ $0 \cdot 10$.

Figure 5 gives the percentage absorption and retention of amino acids and peptide after a 15 -min period of the test solutions within the intestinal lumen. After injection of the amino acid mixture, tryptophan was absorbed about twice as rapidly as glycine. After gly-trp, $43 \%$ of the peptide remained unhydrolysed within the lumen and free tryptophan amounted to $13 \%$ and glycine $33 \%$ of the amounts contained in the injected peptide. Preliminary experiments at a lower concentration of $0.01 \mathrm{~m}-\mathrm{mole} / \mathrm{ml}$, using the method of Young and Edwards (1966) to assess absorption, give a mean of only $8 \%$ peptide remaining at $15 \mathrm{~min}$ with $44 \%$ free tryptophan and $60 \%$ free glycine remaining within the lumen. Incubation of intestinal contents with added peptide in vitro showed that no further hydrolysis of peptide occurred in the centrifuged specimens, but without prior centrifugation there was continued hydrolysis of peptide for 60 minutes. After this period peptide concentration was one half the initial value and hydrolysis after this was negligible. The rate of peptide breakdown in the first 15 min of incubation was, however, only $25 \%$ that of peptide hydrolysis within the gut lumen, indicating that peptidase activity within the intestinal cell wall or at the brush border was quantitatively more important than that contributed by luminal desquamated mucosal cells. Mucosal cells obtained immediately after death contained large amounts of free glycine and tryptophan, but unhydrolysed glycyl-tryptophan was not detectable. Heizer and Laster (1969) have shown that the activity of glycyl-tryptophan hydrolase within rat intestinal mucosal cells is very high.

Results with phenylalanyl-phenylalanine were similar but less free amino acid remained within 
the lumen after 15 min (Fig. 4). The mean was $66 \%$ peptide and $8 \%$ free phenylalanine unabsorbed. Incubation of intestinal contents with added peptide in vitro showed that in uncentrifuged fluid, the rate of hydrolysis was approximately equal to that occurring when peptide was present in the gut lumen ( $8 \%$ after $15 \mathrm{~min})$. In centrifuged fluid, the rate was halved. The results suggest that phenylalanyl-phenylalanine dipeptidase is more soluble in intestinal fluid than glycyl-tryptophan dipeptidase, and that back diffusion of phenylalanine from the mucosal cells is either zero or very low in amount.

\section{Discussion}

The present results confirm and extend data on carnosine ( $\beta$-alanyl-L-histidine) absorption by the same case of Hartnup disease (Navab and Asatoor, 1970). After ingestion of carnosine, serum concentrations of $\beta$-alanine and of histidine were within normal limits, whereas after ingestion of an equimolar mixture of the two constituent amino acids, absorption of histidine was grossly reduced but $\beta$-alanine $a b$ sorption was normal. Halvorsen, Hygstedt, Jagenburg, and Sjaastad (1969) have recently published a study of L-histidine absorption in two cases of Hartnup disease. They found extremely flat tolerance curves for serum histidine comparable to the results of Navab and Asatoor (1970). As neither free histidine nor its bacterial metabolites were detected in the faeces, the results of Halvorsen et al (1969) were interpreted as due to an unduly rapid removal of histidine from the blood stream, both by excess urinary excretion and by an increased uptake of the amino acid by histidine-deficient body cells. The fact that serum histidine concentrations are within normal limits after carnosine ingestion shows that this interpretation must be incorrect. The flat histidine tolerance curve after ingestion of large doses of free histidine in Hartnup disease is largely explained by grossly reduced intestinal absorption of the amino acid. Cases of Hartnup disease show evidence of nutritional defect to be expected in a disease in which most of the essential amino acids are poorly absorbed by the gut. Thus, the mean stature of cases of Hartnup disease is significantly reduced below normal values (Navab and Asatoor, 1970), and the main presenting symptoms and signs of the disease are those of clinical pellagra, due to deficiency of tryptophan and of its metabolite nicotinamide.

Histidine is not an essential amino acid, and therefore it was decided to concentrate on peptides containing essential amino acids known to be poorly transported in Hartnup disease, phenylalanine and tryptophan being the ones selected. The previous demonstration of a normal intestinal transport of carnosine, despite reduced absorption of free histidine, gives only limited information concerning the mechanisms of dipeptide transport by the human gut. $\beta$-Alanine is known to be transported by a different system to that involved in histidine transport both in the gut and in the renal tubule (De la Noüe, Newey, and Smyth, 1969; Scriver, Pueschel, and Davies, 1966). Normal carnosine absorption in Hartnup disease could, therefore, conceivably be explained by entry of the dipeptide into the mucosal cell via the entirely normal $\beta$-alanine sites (Fig. 6). To test this possibility a dipeptide, ie, phenylalanyl-phenylalanine, was selected which consisted entirely of a single amino acid known to be poorly transported in Hartnup disease. Since the dipeptide was considerably better absorbed than an equivalent amount of the free amino acid, it is unlikely that the uptake systems for the two substances in the gut are identical. Dipeptides may possibly be absorbed by an independent system similar to that described in bacteria (Payne, 1968). Transport of the free amino acid is grossly deficient in Hartnup disease, whereas that of the dipeptide is either within normal limits or only very slightly impaired. The results suggest that nutritional requirements of phenylalanine are met in the normal subject by a dual mechanism of transport of both free amino acid and of oligopeptides containing phenylalanine, whereas cases of Hartnup disease have to rely almost entirely on the latter mechanism alone. Unfortunately it was not possible to carry out more than two tolerance tests in normal subjects, and even these were at a lower dosage. One of the normal subjects absorbed both the dipeptide and the free amino acid much more rapidly than did the other, indicating that there is a considerable variation in rates of absorption between different individuals.

Glycyl-L-tryptophan was selected in an attempt to explain the disproportionate nutritional deficiency of tryptophan in Hartnup disease. Any of the other 38 dipeptides containing tryptophan which might be derived from protein hydrolysis could equally well have been used, but glycyl-tryptophan was the only one easily available in bulk supply. The results are similar in principle to those obtained in the cases of carnosine and of phenylalanyl-phenylalanine but differ in detail. As with the other two dipeptides, absorption of the amino acid involved in the transport defect of Hartnup disease was much more efficient from the dipeptide than from the free amino acid itself. Absorption of tryptophan from gly-trp was unequivocally subnormal, whereas that of histidine from carnosine and of phenylalanine from phe-phe was either normal or only minimally reduced. Accurate interpretation of this finding is impossible because of current inadequate knowledge of the complexities of dipeptide transport. Important unanswered questions are as follows. 
2
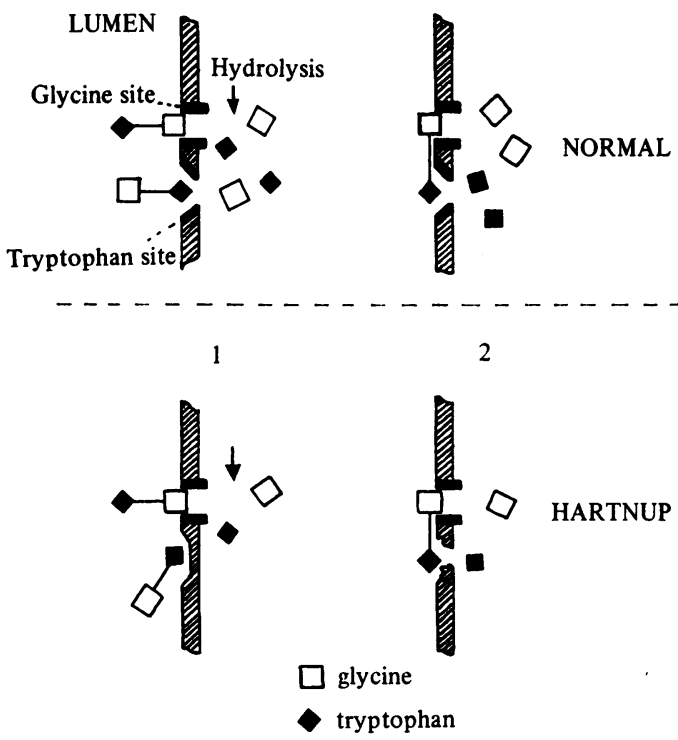

Fig. 6 Two transport schemes to account for absorption of an 'affected' amino acid in Hartnup disease when presented as a mixed dipeptide such as glycyltryptophan. In scheme 1, dipeptides are taken up by amino acid entry sites. In Hartnup disease, though tryptophan entry sites are absent or defective, glycyltryptophan can still be taken up by the intact glycine sites. In scheme 2, based on suggestions made by Parsons (1969), dipeptides are taken up by attachment to amino acid entry sites, attachment and hydrolysis being different aspects of the same process. In Hartnup disease, the tryptophan entry sites are defective, but the firm attachment of the glycine moiety of glycyltryptophan 'plugs in' the tryptophan sufficiently effectively to secure its entry.

Neither scheme would satisfactorily account for uptake of a dipeptide such as phenylalanyl-phenylalanine, made up entirely of an 'affected' amino acid.

Is the low absorption of tryptophan from gly-trp typical of all cases of Hartnup disease, or is it merely due to a fortuitous low rate of tryptophan transport in this particular patient analogous to that found for phenylalanine absorption from phe-phe in one of the normal subjects?

Is gly-trp representative of the 39 tryptophancontaining dipeptides which might be derived from protein hydrolysis, or was it an unfortunate choice of a tryptophan-dipeptide which is disproportionately poorly absorbed in Hartnup disease?

Is the fraction of tryptophan absorbed by normal subjects as oligopeptides usually lower than the fraction absorbed as peptide in the case of other essential amino acids?

The data from the rat experiments indicate that large amounts of both tryptophan and glycine appear within the gut lumen after intraluminal injection of glycyltryptophan. The similar

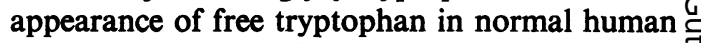
subjects would be of no nutritional significance as it would be rapidly absorbed by the normal $\stackrel{\vec{S}}{+}$ neutral amino acid transport system. By con-o trast, the fraction appearing as free tryptophan would be of negligible nutritional value in a $\frac{\bar{\omega}}{7}$ case of Hartnup disease. The data from this $\stackrel{\mathbb{D}}{\propto}$ patient show that absorption of free tryptophan $\cong$ from the gut is almost zero. Plasma levels of $\vec{a}$ tryptophan after ingestion of the free amino acid barely increased over the fasting value, and $\vec{\omega}$ previous tests (Navab and Asatoor, 1970) have $\stackrel{\odot}{\circ}$ shown that at least $30 \%$ of ingested free trypto- $\stackrel{\varrho}{=}$ phan was recoverable as urinary indican, a derivative which is obviously nutritionally $\overrightarrow{i r}$ valueless. Possibly, therefore, the disproportionate $\dot{\omega}$ tryptophan deficiency in Hartnup disease is due ${ }^{\infty}$ to accumulation of the free amino acid within 음 the gut lumen to a greater fraction of the total $\rightarrow$ tryptophan intake than that found for other 3 essential amino acids. Much more work on peptide transport by the gut must, however, $\vec{\varphi}$ be carried out before this is any more than a 0 hypothesis.

A jejunal biopsy in this patient was histologically normal. Enzyme studies showed that maltase, lactase, and glycyl-glycine dipeptidase activity of the mucosa was within normal limits, but $\frac{}{\Phi}$ peptidase activity against glycyl-leucine and $\stackrel{\circ}{\rightarrow}$ leucyl-glycine was considerably depressed. The low dipeptidase activities might possibly be the result of protein malnutrition (Solimano, Burgess, and Levin, 1967), but it is interesting that the two definitely subnormal activities were against mixed peptides containing an 'affected' amino acid (leucine). This observation could be 3 interpreted in terms of the transport scheme $ᄋ$ suggested by Parsons (1969) (Fig. 6). The ability of the patient to absorb L-phenylalanyl-의 L-phenylalanine better than the free amino $\triangle$ acid, however, indicates that this scheme is unlikely to be valid.

We wish to thank Professor I. M. P. Dawson for examination of the jejunal biopsy, and Dr F. Sadikalic and Miss Maria Lis for the enzyme estimations.

Dr K. D. G. Edwards was in receipt of a grant from ? the research funds of the Westminster Hospital 0 Board of Governors.

References

Alexander, B., Landwehr, G., and Seligman, A. M. (1945). A specific micromethod for the colorimetric determination $\leq$ of glycine in blood and urine. J. biol. Chem., 160, 51-59.

Craft, I. L., Geddes, D., Hyde, C. W., Wise, I. J., and Matthews, ? D. M. (1968). Absorption and malabsorption of glycine? and glycine peptides in man. Gut, 9, 425-437.

De la Noüe, J., Newey, H., and Smyth, D. H. (1969). Transport of alanine isomers by rat small intestine in vitro. J. Physiol. (Lond.), 202, 100-101P. 
Halvorsen, S., Hygstedt, O., Jagenburg, R., and Sjaastad, O. (1969). Cellular transport of L-histidine in Hartnup disease. J. clin. Invest., 48, 1552-1559.

Heizer, W. D., and Laster, L. (1969). Hydrolases in the mucosa of rat small intestine for phenylalanine containing dipeptides. Biochem. biophys. Acta (Amst.), 185, 409-423.

Hsia, D. Y.-Y., Knox, W. E., and Paine, R. S. (1957). A case of phenylketonuria with borderline intelligence. Amer. J. Dis. Childh., 94, 33-39.

Matthews, D. M., Lis, M. T., Cheng, B., and Crampton, R. F. (1969). Observations on the intestinal absorption of some oligopeptides of methionine and glycine in the rat. Clin. Sci., 37, 751-764.

Milne, M. D., Crawford, M. A., Cirao, C. B., and Loughridge, L. W. (1960). The metabolic disorder in Hartnup disease. Quart.J. Med., 29, 407-421.

Navab, F., and Asatoor, A. M. (1970). Studies on intestinal absorption of amino acids and a dipeptide in a case of Hartnup disease. Gut, 11, 373-379.

Newey, H., and Smyth, D. H. (1962). Cellular mechanisms in intestinal transfer of amino acids. J. Physiol. (Lond.), 164, 527-551.

Parsons, D. S. (1969). Black box models of intestinal mucosal function. In International Symposium on Intestinal Transfer of Electrolytes, Amino Acids, and Sugars, edited by W. Armstrong. Thomas, Springfield.

Payne, J. W. (1968). Oligopeptide transport in Escherichia coli. J. biol. Chem., 243, 3395-3403.

Scriver, C. R., Pueschel, S., and Davies, E. (1966). Hyper- $\beta$ alaninemia, associated with $\beta$-aminoaciduria, and $\gamma-$ aminobutyricaciduria, somnolence, and seizures. New Engl.J. Med., 274, 635-643.

Solimano, G., Burgess, E. A., and Levin, B. (1967). Proteincalorie malnutrition: effect of deficient diets on enzyme levels of jejunal mucosa of rats. Brit. J. Nutr., 21, 55-68.

Technicon Instruments Co. (1966). Techniques in Amino Acid Analysis, pp. 104-114. Technicon, Chertsey.

Udenfriend, S., and Peterson, R. E. (1957). Assay of aromatic amino acids: tryptophan. Meth. Enzymol., 3, 613-614.

Young, J. A., and Edwards, K. D. G. (1966). Competition for transport between methyldopa and other amino acids in rat gut loops. Amer. J. Physiol., 210,1130-1136.

Young, J. A., Reid, J., and Edwards, K. D. G. (1964). Amino acid separation and quantitation of amino acids using high voltage paper electrophoresis. Proc. Aust. Ass. clin. Biochem., 1, 95-100.
(1) 